\title{
Detection and correction of patient movement in prostate brachytherapy seed reconstruction
}

\author{
Steve T Lam ${ }^{1}$, Paul S Cho ${ }^{2}$, Robert J Marks II ${ }^{3}$ and Sreeram Narayanan ${ }^{2}$ \\ ${ }^{1}$ The Boeing Company, Seattle, WA 98124-2207, USA \\ ${ }^{2}$ Department of Radiation Oncology, University of Washington, Box 356043, Seattle, \\ WA 98195-6043, USA \\ ${ }^{3}$ Department of Engineering, Baylor University, Waco, TX 76798-7356, USA \\ E-mail: psncho@u.washington.edu
}

Received 12 January 2005, in final form 13 March 2005

Published 20 April 2005

Online at stacks.iop.org/PMB/50/2071

\begin{abstract}
Intraoperative dosimetry of prostate brachytherapy can help optimize the dose distribution and potentially improve clinical outcome. Evaluation of dose distribution during the seed implant procedure requires the knowledge of 3D seed coordinates. Fluoroscopy-based seed localization is a viable option. From three x-ray projections obtained at different gantry angles, 3D seed positions can be determined. However, when local anaesthesia is used for prostate brachytherapy, the patient movement during fluoroscopy image capture becomes a practical problem. If uncorrected, the errors introduced by patient motion between image captures would cause seed mismatches. Subsequently, the seed reconstruction algorithm would either fail to reconstruct or yield erroneous results. We have developed an algorithm that permits detection and correction of patient movement that may occur between fluoroscopy image captures. The patient movement is decomposed into translational shifts along the tabletop and rotation about an axis perpendicular to the tabletop. The property of spatial invariance of the co-planar imaging geometry is used for lateral movement correction. Cranio-caudal movement is corrected by analysing the perspective invariance along the $\mathrm{x}$-ray axis. Rotation is estimated by an iterative method. The method can detect and correct for the range of patient movement commonly seen in the clinical environment. The algorithm has been implemented for routine clinical use as the preprocessing step for seed reconstruction.
\end{abstract}

\section{Introduction}

Recent years have seen a resurgence in brachytherapy. In particular, permanent implantation of radioactive seeds has gained popularity as an effective treatment modality for early stage prostate carcinoma (Blasko et al 1987, 1996, Grimm et al 2001). An improvement in the seed 
placement technique aided by image guidance has played a major role in the success of prostate brachytherapy. Further improvement in implant quality is expected through implementation of intraoperative dosimetry. Fluoroscopy-based dosimetry is an attractive candidate due to its real-time imaging capability and availability in the operating room. After the seed positions have been determined from the fluoroscopy projections, various techniques can be used to reconstruct the 3D seed coordinates (Amols and Rosen 1981, Rosenthal and Nath 1983, Biggs and Kelly 1983, Altschuler et al 1983, Jackson 1983, Siddon and Chin 1985, Altschuler and Kassaee 1997, Tubic et al 2001, Narayanan et al 2002, 2004, Todor et al 2003, Lam et al 2004). However, the problem of patient movement that may occur during image acquisition has not been fully addressed. Amols and Rosen (1981) suggested a method to correct for patient motion between films. The authors discussed correction in the $Y$-direction only, which is parallel to the axis of gantry rotation. The method uses the average $Y$-coordinate values of the seeds on each of the three films. Theoretically, because the imaging geometry is co-planar these averages should be the same for all three films. However, the performance evaluation of the method was not presented. Tubic et al (2001) proposed an algorithm using the simulated annealing technique, which has integrated the motion correction in the seed reconstruction process. They have demonstrated motion correction of up to $5 \mathrm{~mm}$ translation and recommended keeping the rotational errors within $\pm 2.5^{\circ}$.

The problem of patient movement may not be an issue under the condition of general anaesthesia. However, for those clinics such as ours that use local anaesthesia for prostate brachytherapy (Wallner et al 1999, Smathers et al 2000, Wallner 2002), patient movement during fluoroscopy image capture becomes a practical problem. If uncorrected, the errors introduced by patient motion between image captures would cause seed mismatches. Subsequently, the seed reconstruction algorithm would either fail to reconstruct or yield erroneous results. We present an algorithm that permits detection and correction of patient movement that may occur between fluoroscopy image captures. The algorithm has been implemented for routine clinical use as the preprocessing step for seed reconstruction.

\section{Methods}

Since the patient is in dorsal lithotomy position, the direction of the body movement is confined to the surface of the patient couch. Therefore, in the patient coordinate system (figure 1), the movement can be decomposed into $X$-translation (cranial-caudal), $Y$-translation (lateral), and rotation about an axis parallel to the $Z$-axis. Correction for each of the movement vectors will be addressed separately. A three-view seed reconstruction technique is assumed.

\subsection{Coordinate systems}

The coordinate system is defined in figure 1 . The reference coordinate system, in which the $3 \mathrm{D}$ seed positions are ultimately defined, is given by $(X, Y, Z)$. The $\mathrm{x}$-ray source is located at $Z$ and rotates about the $Y$-axis. The imaging orientations are anterior-posterior and two anterior obliques angled in the $X$ - (cranio-caudal) direction. The seed coordinates at any X-ray gantry angle other than the reference position are designated as $\left(X^{\prime}, Y^{\prime}, Z^{\prime}\right)$ which relate to $(X, Y, Z)$ according to

$$
\left[\begin{array}{l}
X \\
Y \\
Z
\end{array}\right]=\left[\begin{array}{ccc}
\cos \theta & 0 & \sin \theta \\
0 & 1 & 0 \\
-\sin \theta & 0 & \cos \theta
\end{array}\right]\left[\begin{array}{l}
X^{\prime} \\
Y^{\prime} \\
Z^{\prime}
\end{array}\right]
$$

where $\theta$ is the $\mathrm{X}$-ray gantry angle relative to the reference coordinate system. 


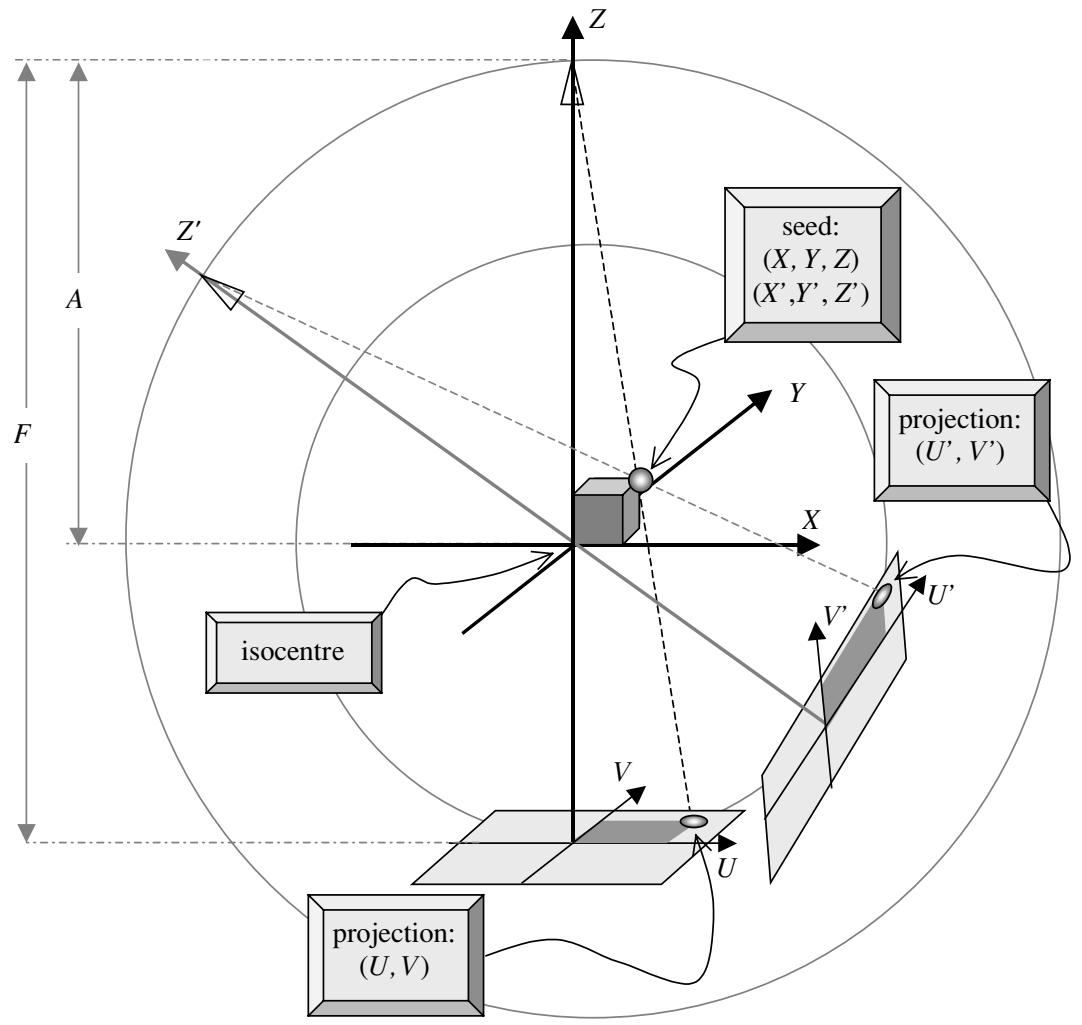

Figure 1. Geometry of seed projections onto two planes. The x-ray source, located at $Z$, rotates about the $Y$-axis through angle $\alpha .(X, Y, Z)$ is the reference and $\left(X^{\prime}, Y^{\prime}, Z^{\prime}\right)$ is the rotated 3D coordinate system. Corresponding projection domains are designated as $(U, V)$ and $\left(U^{\prime}, V^{\prime}\right)$, respectively. $F$ is the distance from the x-ray source to the detector plane and $A$ is the distance from the $\mathrm{x}$-ray source to the isocentre. The dots indicate the location of the seed in the $3 \mathrm{D}$ and projected coordinate systems.

The relationship between the reference patient space $(X, Y, Z)$ and its projection onto detector domain $(U, V)$ is given by

$$
\begin{aligned}
& U=\frac{F X}{A+Z} \\
& V=\frac{F Y}{A+Z}
\end{aligned}
$$

where $F$ is the distance between the x-ray source and the detector and $A$ is the distance between the source and the axis of rotation.

\subsection{Correction for movement in $Y$-direction}

Consider figure 2 in which plane $L$ hinges about the line connecting the two source positions $S_{1}$ and $S_{2}$. There are numerous such planes passing through the implant volume. Every such plane separates the seeds in $3 \mathrm{D}$ and also in projection space in the same manner. To illustrate this, let the intersection of the plane $L$ with the detector planes $I_{1}$ and $I_{2}$ be $\ell_{1}$ and $\ell_{2}$. Note that the black seeds are behind plane $L$ and the white seeds are in front. This same grouping 


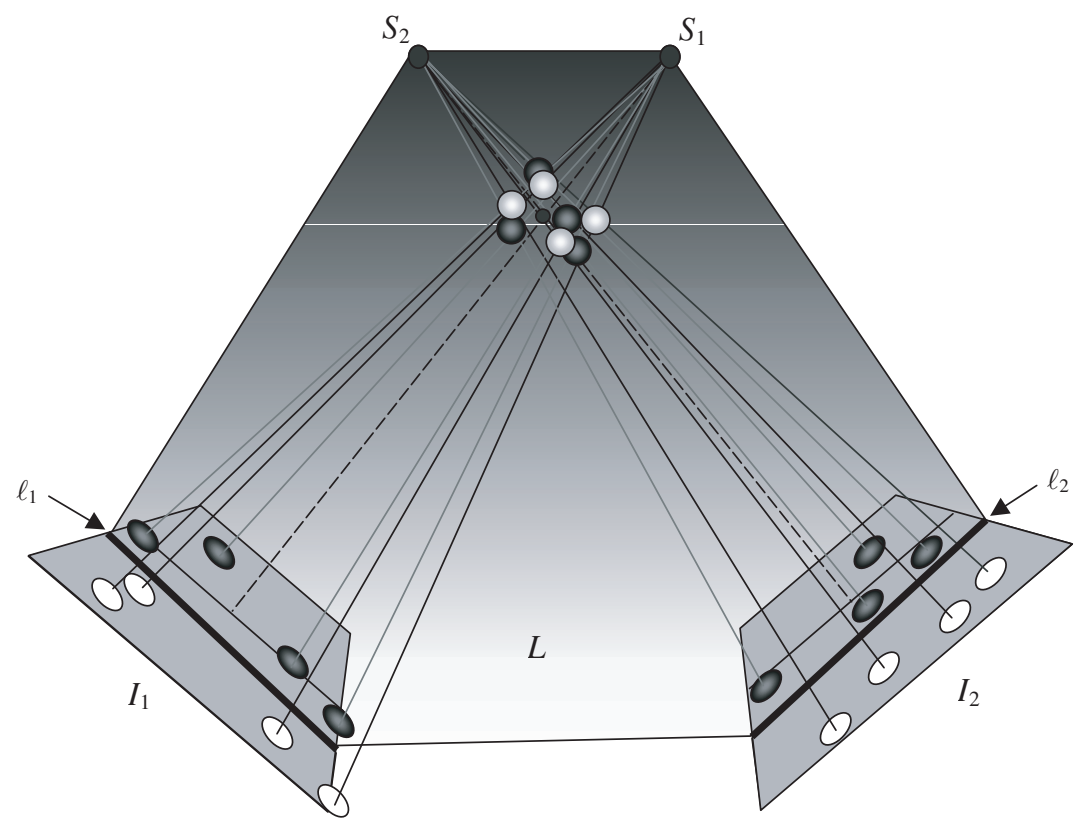

Figure 2. Geometry of eigen line. The x-ray sources are located at $S_{1}$ and $S_{2}$. Plane $L$ hinges on the line connecting $S_{1}$ and $S_{2}$. The dashed lines originating at the sources indicate the central x-ray axis. The black seeds are behind plane $L$ and the white seeds are in front. The plane $L$ intersects the detector planes $I_{1}$ and $I_{2}$ at the lines $\ell_{1}$ and $\ell_{2}$. These lines, called eigen lines, preserve the same grouping of the seeds both in $3 \mathrm{D}$ and $2 \mathrm{D}$ detector planes.

of seeds is preserved as the seeds are projected onto the detector plane. For example, in view $I_{1}$ seed projections shown in black are on one side of line $\ell_{1}$ and white on the other. Furthermore, it is observed that plane $L$ divides the seeds in the same proportion for views $I_{1}$ and $I_{2}$. Therefore, if there is no patient movement between $I_{1}$ and $I_{2}$, the ratio of the seeds in two groups divided by plane $L$ and associated line intersections $\ell_{1}$ and $\ell_{2}$ should remain the same. These lines defined by intersection of $L$ and detector planes will be referred to as 'eigen' lines since they divide the seeds projected at two different gantry angles by the same ratio. The ratio invariant property of an eigen line is used to detect and correct for patient movement in the $Y$-direction as described below.

The equation for plane $L$ with normal vector $\vec{n}_{1}=\left(a_{1}, b_{1}, c_{1}\right)$ is given by

$$
a_{1} x+b_{1} y+c_{1} z+d_{1}=0
$$

where $a_{1}, b_{1}, c_{1}$ and $d_{1}$ are constants, and $a_{1}, b_{1}, c_{1}$ are not all zeros. Similarly, the detector plane can be expressed in a general form of the plane equation with normal vector $\vec{n}_{2}=\left(a_{2}, b_{2}, c_{2}\right)$. The intersection of these two planes defines an eigen line $\vec{l}=(a, b)$, which is given by the cross product

$$
\vec{l}=\vec{n}_{1} \times \vec{n}_{2} .
$$

For an $i$ th seed with a $3 \mathrm{D}$ coordinate $P_{i}=\left(x_{i}, y_{i}, z_{i}\right)$ and projection $Q_{i}=\left(U_{i}, V_{i}\right)$, the eigen line $l_{i}$ is given by the following parametric equations:

$$
\begin{aligned}
& U=U_{i}+t a \\
& V=V_{i}+t b
\end{aligned}
$$

where $-\infty<t<+\infty$. 
Table 1. Rules to determine which view(s) must to be corrected for the $Y$ patient movements.

\begin{tabular}{llll}
\hline \multicolumn{3}{c}{ Seed-count error conditions } & View to be \\
\cline { 1 - 3 } $\begin{array}{llll}\text { Errors between } \\
\text { views 1 and 3 }\end{array}$ & $\begin{array}{l}\text { Errors between } \\
\text { views 1 and 2 }\end{array}$ & $\begin{array}{l}\text { Errors between } \\
\text { views 2 and 3 }\end{array}$ & $\begin{array}{l}\text { corrected for } \\
\text { movement }\end{array}$ \\
\hline$<\lambda$ & $<\lambda$ & $<\lambda$ & None \\
$>\lambda$ & $>\lambda$ & $<\lambda$ & View 1 \\
$<\lambda$ & $>\lambda$ & $>\lambda$ & View 2 \\
$>\lambda$ & $<\lambda$ & $>\lambda$ & View 3 \\
$>\lambda$ & $>\lambda$ & $>\lambda$ & Two of three views \\
\hline
\end{tabular}

For each view, the seeds are ordered by their $V$ values. Under ideal conditions with infinite detector resolution, noiseless and perfect geometry, a single eigen line should be able to detect patient movement in the $Y$-direction. If the ratio of seed counts above and below the eigen line is not identical between the projections, it indicates that the patient has moved.

In practice, however, there are noises in the data arising from pixel error associated with the seed segmentation process, undetected seeds and false positive seeds. These noises could mimic patient movement by reporting unequal seed ratios. Therefore, reliance on only a single eigen line would not be sufficient to determine the true patient movement. For noisy clinical data, it is advantageous to apply the principle of eigen line for each seed. One can tally the number of seeds appearing on one side of the eigen line computed by equations $(6 a)$ and $(6 b)$. We opted to use the seed counts above an eigen line (as opposed to below, which should work just as well). The seed count given by an eigen line intersecting an ith seed on view 1 is denoted by $H_{i}^{1}$. The same eigen line will divide the seeds on view 2 and yield a seed count $H_{i}^{2}$. The seed counts above the eigen line are computed for all eigen lines for each pair of projections. The patient movement is assessed using the average errors in seed counts between projections. The average discrepancy in seed counts between projections 1 and 2 is given by

$$
\bar{H}^{1,2}=\frac{\sum_{i=1}^{N_{1}}\left\|H_{i}^{1}-H_{i}^{2}\right\|}{\left(\frac{N_{1}+N_{2}}{2}\right)}
$$

where $N_{1}$ and $N_{2}$ are the total number of detected seeds in views 1 and 2, respectively. Note that $N_{1}$ and $N_{2}$ are not necessarily the same because some seeds may overlap at a certain projection angle and not be detected (Narayanan et al 2004). The upper limit of summation can be either one of the total seed counts. In addition to the projection pair $\{1,2\}$, the average seed-count errors are calculated for the projection pairs $\{2,3\}$ and $\{1,3\}$ as well.

From the average seed-count errors, one can determine which projection is associated with the patient movement. Patient movement could occur between image captures for views 1 and 2 and/or between views 2 and 3. Table 1 describes the rules to determine which view(s) should be corrected depending on the movement scenario detected by the eigen-line seed-count error analysis. If the average seed-count errors (equation (7)) are above a certain threshold, $\lambda$, it is considered that movement has occurred. The threshold $\lambda$, which is a function of seed density and noise in the data, is determined empirically. The correction can be made by properly aligning the views in the $V$-direction. Here, we describe the general case, in which multiple views (i.e., two out of three views) need to be corrected.

If there are patient movements between image captures, one of the views is kept unchanged and the relative positions of the other two views are estimated. For the best $3 \mathrm{D}$ reconstruction results, the total correction distance on the non-reference views must be minimized. This is accomplished as follows: 
1. From the average seed count errors $\bar{H}^{1,2}, \bar{H}^{2,3}$ and $\bar{H}^{1,3}$ determine which view-pair has the maximum value.

2. Select a view that is not associated with the maximum average seed count error. For example, given the errors $\bar{H}^{1,2}=2, \bar{H}^{2,3}=1$ and $\bar{H}^{1,3}=3$, the error for $\bar{H}^{1,3}$ is the largest. Consequently, view 2 is selected as the reference, i.e., keep this view stationary.

3. Calculate the correction for the other views, i.e., 1 and 3:

(1) Calculate the average of the $V$ seed coordinates of all three views 1,2 and $3\left(V_{1}, V_{2}\right.$ and $\left.V_{3}\right)$.

(2) Calculate the error for view 1 with respect to the reference view, i.e., $V_{2}-V_{1}$.

(3) Likewise, calculate the error for view 3, i.e., $V_{2}-V_{3}$.

4. Keeping the reference view stationary, shift the other two views by the respective calculated errors.

The $V$-coordinate correction is an approximation of the patient movement in the $3 \mathrm{D} Y$ coordinates but works well for the range of patient movement that is typical during the brachytherapy implant procedure.

\subsection{Correction for rotational movement}

Rotational movement about an axis parallel to the $Z$-axis will also cause seed-count mismatches among the views. Therefore, if the movement includes the rotational component, the errors in the eigen-line-based seed counts will remain appreciable even after the $Y$-movement correction is made. This is because the rotation will cause the $Y$ component of the projection between the views not to line up exactly. The same rules for the $Y$-movement correction (table 1) are used to identify the view(s) that need rotational adjustment.

The patient rotation about an axis passing through $\left(X_{c}, Y_{c}\right)$ that is parallel to the $Z$-axis is given by

$$
\left[\begin{array}{l}
X \\
Y \\
Z
\end{array}\right]=\left[\begin{array}{ccc}
\cos \varsigma & -\sin \varsigma & 0 \\
\sin \varsigma & \cos \varsigma & 0 \\
0 & 0 & 1
\end{array}\right]\left[\begin{array}{c}
X^{\prime}-X_{c} \\
Y^{\prime}-Y_{c} \\
Z^{\prime}
\end{array}\right]
$$

where $\varsigma$ is the rotational angle. There is no analytical solution for the values of $\left(X_{c}, Y_{c}\right)$ and $\varsigma$. Therefore, iterative corrections are applied to suspected view(s) by changing the centre of rotation and the angle. The correction process is repeated until the average error of the eigen-line-based seed-count errors is minimized. In order to speed up the iterative process, a quadtree search algorithm is used. The quadtree algorithm, illustrated in figure 3 , is a recursive, near-neighbour range search, where the range is in a region of a plane. The plane is partitioned into four equal quadrants. In our application, the search is performed along the orthogonal axis of the starting root point with a given range. The subsequent starting root is the best result from the previous search. When a search does not return an improved result, the search range from the current root is reduced to further refine the search. The algorithm is repeated until the search range distance is less than a given criterion, which in our case is $1 \mathrm{~mm}$.

\subsection{Correction for movement in X-direction}

The $X$-component of the patient movement can be detected by examining the $Z$-component as illustrated in figure 4 , which is drawn on the plane defined by the co-planar $X$-ray sources and the isocentre. $X$-movement analysis is made by collapsing the $3 \mathrm{D}$ imaging geometry onto this plane. The $Z$-axis for each projection lies on the plane. For demonstration purposes, we 


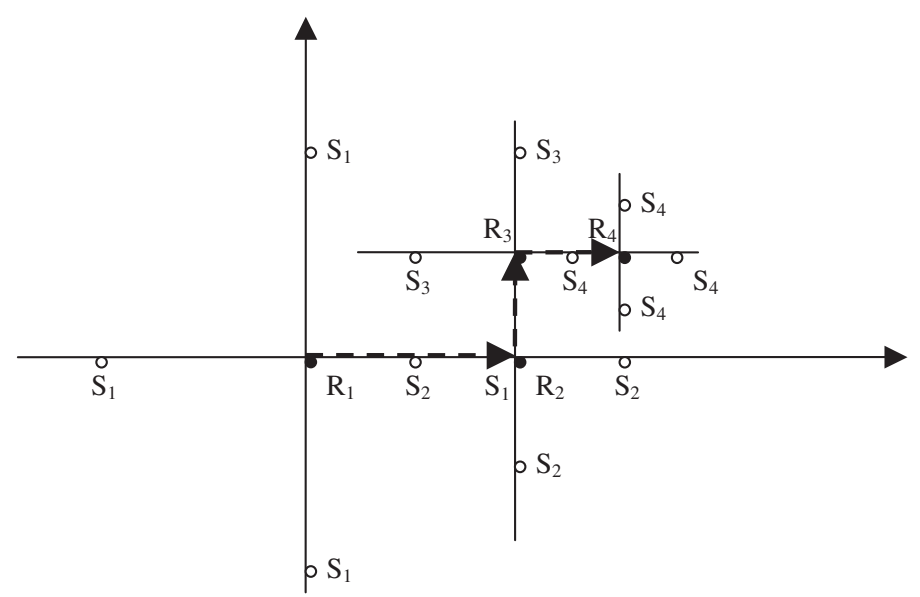

Figure 3. A fast search method for the most likely position of the rotational axis is illustrated. The plane parallel to the tabletop is partitioned into four equal quadrants. The search is performed along the orthogonal axis of the starting root point, $R_{1}$, with a given search range bounded by $S_{1}$. The subsequent starting root is the best result from the previous search. For example, if $S_{1}$ on the right yields the best result, it is set as the new root $R_{2}$. When a search does not return an improved result, the search range associated with the current root is reduced to refine the search. This process is repeated for $R_{3}$ and so on.

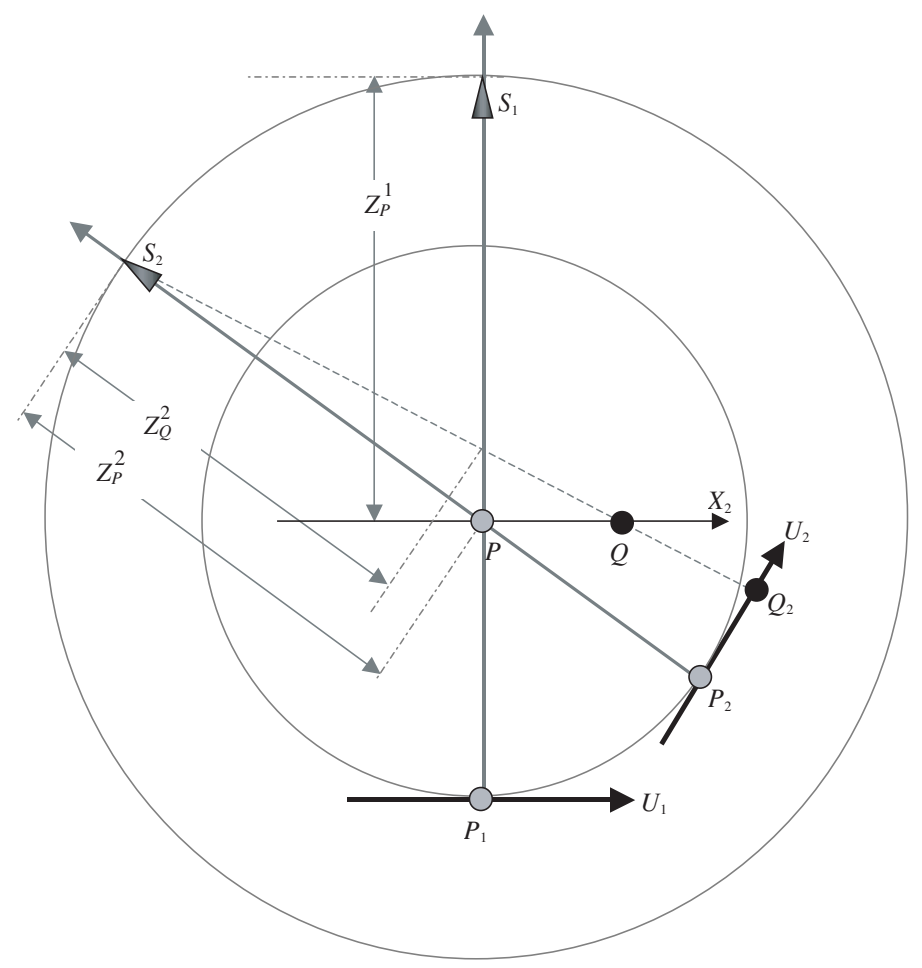

Figure 4. Geometry of $X$ correction. The $Z$ value should remain the same if there is no patient movement in the $X$-direction. 
simplify the diagram by placing a reference seed point $P$ at the centre of rotation. Note that the seed position is determined by the intersection of the rays connecting the $\mathrm{x}$-ray source and the projected seed position on the detector. For example, the seed position $P$ is at the intersection of rays $P_{1} \rightarrow S_{1}$ and $P_{2} \rightarrow S_{2}$.

As shown, the patient movement in the $X$-direction manifests itself in a $Z$-coordinate change. For example, in the absence of patient movement, the $Z$-coordinate of point $P$ will remain the same for views 1 and 2, i.e., $Z_{P}^{1}=Z_{P}^{2}$. (The superscript denotes the different projection angles.) Now let us assume that the patient has moved from $P$ (captured in view 1 ) to $Q$ (captured in view 2). As can be seen, the $Z$ values of the calculated seed position are no longer the same, i.e., $Z_{P}^{1}>Z_{Q}^{2}$. Therefore, the calculated $Z$ values for different views should remain unchanged if there is no $X$-component in the patient's movement. Likewise, the presence of $X$-movement associated with the third view can be detected.

Since it is unknown how individual seeds from different views correspond with one another, the projected seed centroids are used for the $X$-movement analysis. This is a reasonable method, as the implanted seeds will most likely maintain a constant spatial relationship among themselves when the patient moves. From a given view, the $U$-coordinate of the seed centroid is calculated as follows:

$$
\bar{U}=\frac{1}{N} \sum_{i=1}^{N} U_{i}
$$

where $N$ is the number of seeds captured in the view. Given the centroids for views 1, 2 and 3 we can calculate the $Z$ values of the centroids. Two views are required to determine the $Z$ value and this is done by computing the intersection of the lines connecting each $U$ centroid and the respective $\mathrm{x}$-ray source. Let $Z^{1,2}$ denote the reconstructed $Z$ value for views 1 and 2 , and similarly $Z^{1,3}$ and $Z^{2,3}$. Also, let the x-ray projection angles for views 1,2 and 3 be $\theta$, $\theta+\alpha$ and $\theta-\beta$. Using the knowledge of imaging geometry and coordinate transformation between views, the $Z$ values can be calculated as follows:

$$
\begin{aligned}
Z^{1,2} & =\frac{B \bar{U}_{\theta+\alpha} A-C \bar{U}_{\theta} A}{C \bar{U}_{\theta} \cos \theta+C F \sin \theta-B \bar{U}_{\theta+\alpha} \cos (\theta+\alpha)-B F \sin (\theta+\alpha)} \\
Z^{1,3} & =\frac{B \bar{U}_{\theta-\beta} A-D \bar{U}_{\theta} A}{D \bar{U}_{\theta} \cos \theta+D F \sin \theta-B \bar{U}_{\theta-\beta} \cos (\theta-\beta)-B F \sin (\theta-\beta)} \\
Z^{2,3} & =\frac{C \bar{U}_{\theta-\beta} A-D \bar{U}_{\theta+\alpha} A}{D \bar{U}_{\theta+\alpha} \cos (\theta+\alpha)+D F \sin (\theta+\alpha)-C \bar{U}_{\theta-\beta} \cos (\theta-\beta)-C F \sin (\theta-\beta)}
\end{aligned}
$$

where $A$ is the distance from the $\mathrm{x}$-ray source to the axis of rotation, $F$ is the source-to-detector distance, and

$$
\begin{aligned}
& B=F \cos \theta-\bar{U}_{\theta} \sin \theta \\
& C=F \cos (\theta+\alpha)-\bar{U}_{\theta} \sin (\theta+\alpha) \\
& D=F \cos (\theta-\beta)-\bar{U}_{\theta} \sin (\theta-\beta) .
\end{aligned}
$$

If $Z^{1,2}, Z^{1,3}$ and $Z^{2,3}$ are not the same, patient movement exists and needs to be corrected for. The correction strategy is to translate the views in the $U$-direction until the corresponding $Z$ values for all three view-pairs become the same. Since the correction is an approximate 


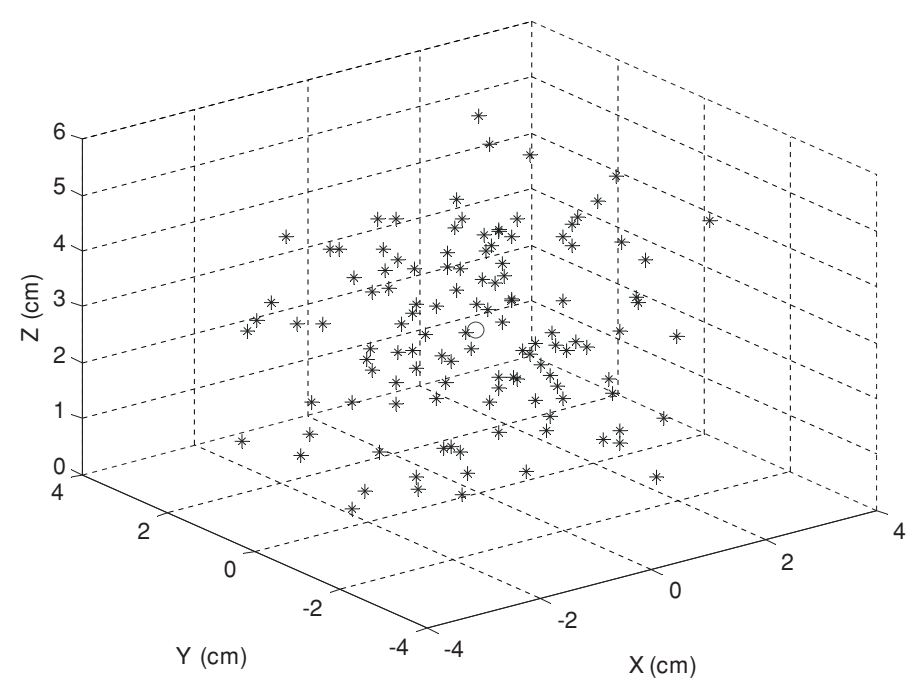

Figure 5. Distribution of the simulation data (the circle indicates the centroid of the distribution).

method performed in 2D space for a 3D problem, it is best to minimize the total correctional translation in the $U$-direction. The following steps accomplish this:

(1) Select a view as a reference. Calculate the total $U$ translation distance needed to align the other two views to the reference view. Call the total correction distance $\Delta U 1$.

(2) Select other views as a reference and repeat the process. Calculate $\Delta U 2$ and $\Delta U 3$.

(3) Choose the view with the minimum $\Delta U$ as the reference for movement correction.

Thus, the determined reference view is fixed and the other two views are translated in the $U$-direction so that the $Z$ values are equal for all three view-pairs.

\section{Results}

Simulation and empirical data were used to verify the method. Examples of clinical cases are also presented.

\subsection{Simulation study}

For the simulation study 120 seed centroids were randomly generated within a $5 \mathrm{~cm}$ cube centred about the isocentre. A $5 \mathrm{~cm}$ cube should enclose most prostates. Figure 5 shows the distribution of the simulation data. With the simulation data, the truth (the $3 \mathrm{D}$ seed coordinates and patient movement) is known. Therefore, the effectiveness of the algorithm can be accurately evaluated against the simulation data. $Y$ correction was followed by incremental rotational correction. Then $X$ correction was applied at the end. Three projections of the seed centroids were generated at $15^{\circ}$ increments from $165^{\circ}$ to $195^{\circ}$. The gantry angle of $180^{\circ}$ indicates the anterior-posterior or the vertical direction. The source-to-axis distance and the source-to-detector distance were set to $100 \mathrm{~cm}$ and $140 \mathrm{~cm}$, respectively, which are similar to the imaging geometry of our fluoroscopy unit. After generating the $165^{\circ}$ and $180^{\circ}$ projection data, the digital phantom was moved in $X$ - and $Y$-directions and was also rotated by various amounts. After the simulated patient movement the $195^{\circ}$ projection was generated. Since 
Table 2. Variables associated with the simulation of patient movement.

\begin{tabular}{|c|c|c|c|c|}
\hline \multirow{2}{*}{$\begin{array}{l}\text { Case } \\
\text { number }\end{array}$} & \multicolumn{2}{|c|}{ Translation $(\mathrm{cm})$} & \multirow{2}{*}{$\begin{array}{l}\text { Rotation } \\
\left({ }^{\circ}\right)\end{array}$} & \multirow{2}{*}{$\begin{array}{l}\text { Rotation axis } \\
\text { offset from } \\
Z \text {-axis }(\mathrm{cm})\end{array}$} \\
\hline & $X$ & $Y$ & & \\
\hline 1 & 1 & 1 & 3 & 0 \\
\hline 2 & 1 & 1 & 3 & 15 \\
\hline 3 & 2 & 2 & 3 & 0 \\
\hline 4 & 2 & 2 & 3 & 15 \\
\hline 5 & 3 & 3 & 3 & 0 \\
\hline 6 & 3 & 3 & 3 & 15 \\
\hline
\end{tabular}

Table 3. Seed reconstruction accuracy for the simulation study.

\begin{tabular}{llllllll}
\hline \multirow{2}{*}{$\begin{array}{l}\text { Case } \\
\text { number }\end{array}$} & \multicolumn{2}{l}{ RMS error $(\mathrm{mm})$} & & \multicolumn{3}{c}{ Maximum error $(\mathrm{mm})$} \\
\cline { 2 - 3 } \cline { 6 - 7 } & $X$ & $Y$ & $Z$ & & $X$ & $Y$ & $Z$ \\
\hline 1 & 0.1 & 0.1 & 0.5 & & 0.5 & 0.4 & 1.9 \\
2 & 0.1 & 0.2 & 0.5 & & 0.9 & 0.6 & 2.5 \\
3 & 0.1 & 0.1 & 0.7 & & 0.8 & 0.6 & 2.7 \\
4 & 0.1 & 0.2 & 0.6 & & 0.9 & 0.6 & 2.8 \\
5 & 0.2 & 0.2 & 0.7 & 0.9 & 0.8 & 2.9 \\
6 & 0.2 & 0.2 & 0.7 & 0.5 & 0.6 & 2.8 \\
\hline
\end{tabular}

the rotational axis is not necessarily at the centre of the prostate ( $Z$-axis), the axis position relative to the $Z$-axis was varied from 0 to $15 \mathrm{~cm}$ along the cranio-caudal ( $X$-axis) direction. Seed displacement due to rotational movement will increase with the rotational-axis offset. In addition, in order to simulate the seed localization errors, Gaussian noise $(\sigma=0.487 \mathrm{~mm}$, which is equivalent to one pixel of our imaging system) was added to all the projections. Table 2 summarizes the parameters associated with the movement simulation studies.

For each simulation case, the movement correction algorithm was applied to the projection data. Subsequently, the 3D seed coordinates were computed using our seed reconstruction algorithm (Narayanan et al 2002, 2004). The results were compared with the known seed coordinates and errors were quantified in terms of RMS and maximum errors as shown in table 3 .

\subsection{Phantom study}

The algorithm was also tested using empirical data. A prostate phantom was implanted with 61 non-radioactive I-125 seeds and the projection data were obtained with a fluoroscopy unit. The source-to-axis distance and the source-to-detector distance were set to $100 \mathrm{~cm}$ and $140 \mathrm{~cm}$, respectively. The centre of the prostate phantom was placed near the isocentre and fluoroscopy images were acquired using the 6-inch mode of the image intensifier. Three views were captured at $20^{\circ}$ increments from $160^{\circ}$ to $200^{\circ}$. First, without moving the phantom, projection images were acquired at three different angles. Later 3D reconstruction was performed from this data set, which constituted the truth. Next, the phantom was moved in $X-Y$-directions and was also rotated as described in table 4. Imaging was then repeated for the $160^{\circ}$ orientation. The projection images were corrected for spatial distortion due to the image intensifier, and the seed centroids were automatically detected (Cho 2000). Subsequently, 

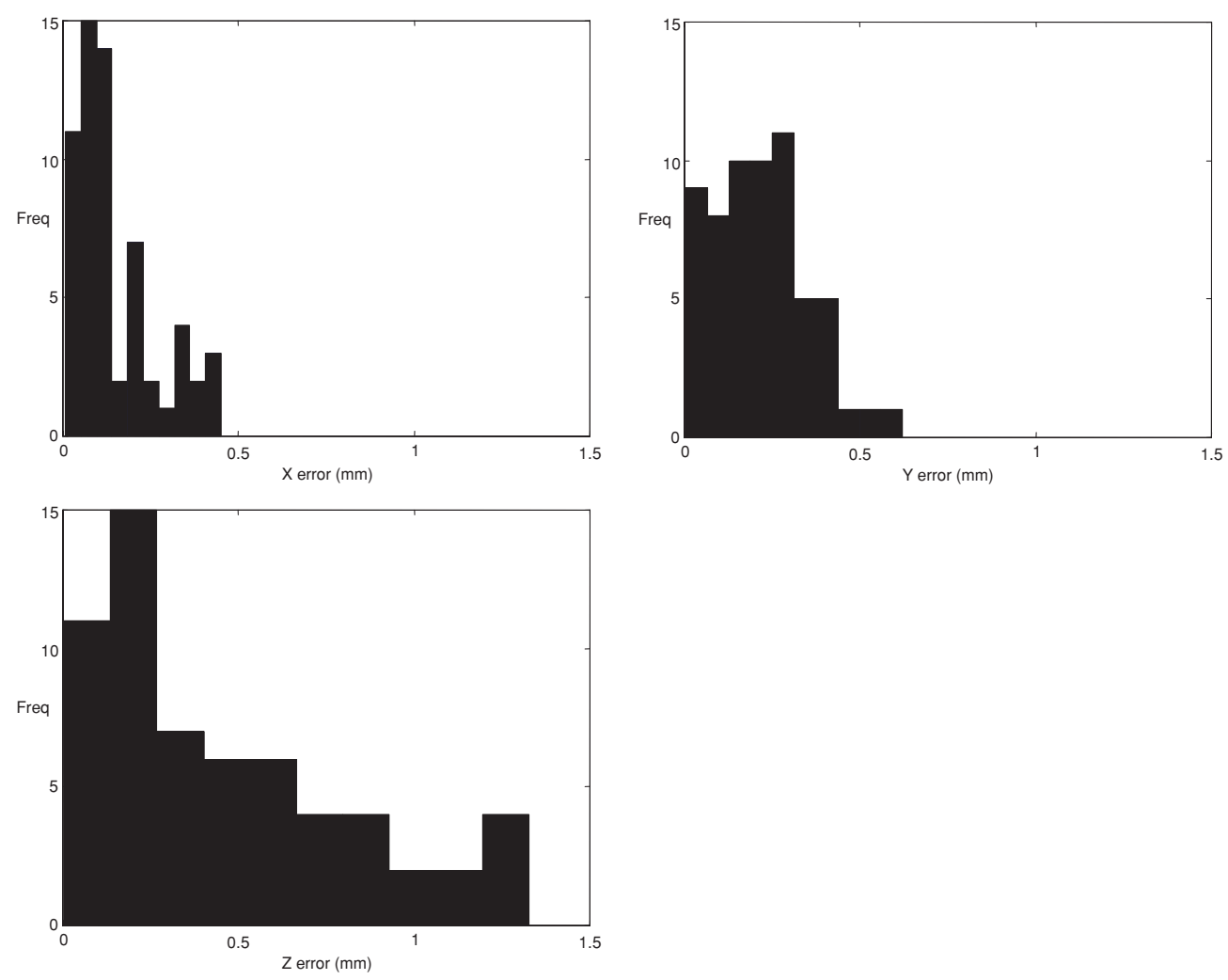

Figure 6. Histograms of $X, Y, Z$ errors for the phantom study.

Table 4. Movement variables for the physical phantom study.

\begin{tabular}{|c|c|c|c|}
\hline \multicolumn{2}{|c|}{ Translation $(\mathrm{cm})$} & \multirow{2}{*}{$\begin{array}{l}\text { Rotation } \\
\left({ }^{\circ}\right)\end{array}$} & \multirow{2}{*}{$\begin{array}{l}\text { Rotation axis offset } \\
\text { from } Z \text {-axis }(\mathrm{cm})\end{array}$} \\
\hline$X$ & $Y$ & & \\
\hline 2 & 2 & 3 & 10 (along $X$ ) \\
\hline
\end{tabular}

Table 5. Seed reconstruction accuracy for the phantom study.

\begin{tabular}{lllllll}
\hline \multicolumn{3}{c}{ RMS error (mm) } & & \multicolumn{3}{c}{ Maximum error (mm) } \\
\cline { 1 - 2 } \cline { 5 - 7 }$X$ & $Y$ & $Z$ & & $X$ & $Y$ & $Z$ \\
\hline 0.2 & 0.3 & 0.6 & & 0.5 & 0.6 & 1.3 \\
\hline
\end{tabular}

the movement correction algorithm was applied and 3D seed reconstruction performed. The results of seed reconstruction involving the movement were compared with the truth. RMS and maximum errors are reported in table 5. Histograms of errors in $X-, Y$ - and $Z$-directions are also shown in figure 6 . In addition, the reconstructed 3D seed coordinates were projected onto the fluoroscopy data in order to visualize the effectiveness of the algorithm (figure 7). 


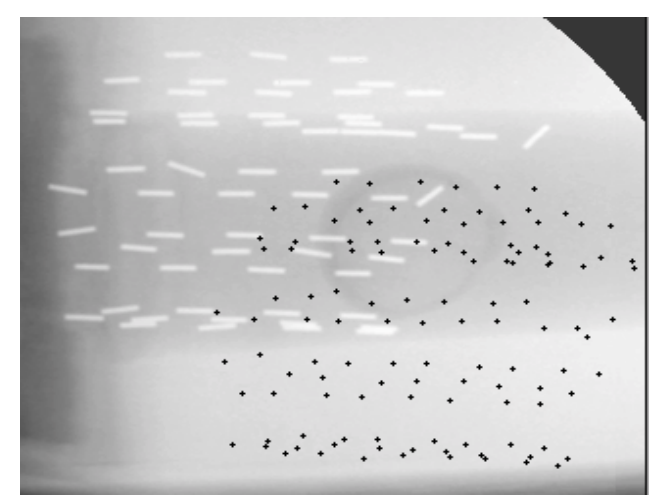

$160^{\circ}$

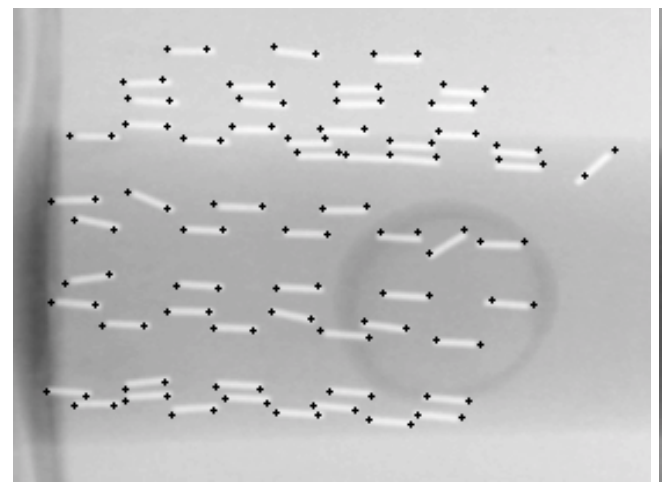

$180^{\circ}$

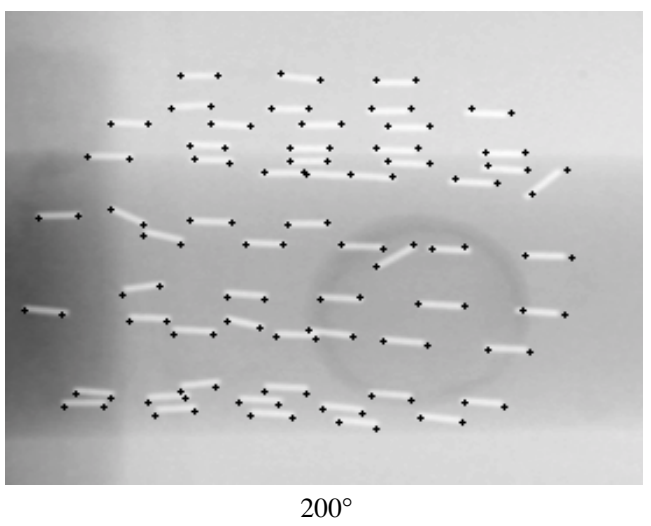

$200^{\circ}$

Figure 7. Fluoroscopic images for the phantom study: $160^{\circ}, 180^{\circ}$ and $200^{\circ}$ views. The projection seed data were corrected for movement and 3D reconstruction performed. The resultant 3D seed end-points were forward projected (black dots).

\subsection{Clinical examples}

Figure 8(a) shows an example of seed reconstruction involving patient movement. As can be seen, without the motion correction the reconstruction algorithm could not reconstruct the seeds accurately. The resultant 3D seed coordinates were forward projected onto the $180^{\circ}$ view demonstrating incomplete, erroneous reconstruction. Figure 8(b) shows the reconstruction results with motion correction. The reconstructed seed centroids were forward projected onto each view demonstrating satisfactory reconstruction as verified on $160^{\circ}$ and $180^{\circ}$ views. The movement occurred between image captures for $180^{\circ}$ and $196^{\circ}$ angles. The movement was estimated to be $2.50 \mathrm{~cm}$ and $2.41 \mathrm{~cm}$ in $X$ - and $Y$-directions, respectively. No rotation was detected.

Figure 9 shows another clinical example. In this case, the patient moved twice; between first and second image capture and again between second and third. The first view $\left(171^{\circ}\right.$ projection) was chosen as the reference and the other two views were corrected for patient movement. Seed reconstruction was performed on the corrected data set and the results were forward projected onto the original seed images. The projected seed centroids coincide with those of the reference fluoroscopy image $\left(171^{\circ}\right.$ projection). Projections onto the other two views indicate that the centroid configurations correspond to the seed images with translational offset. Reconstructed seed coordinates were registered with the CT-derived seed coordinates. 


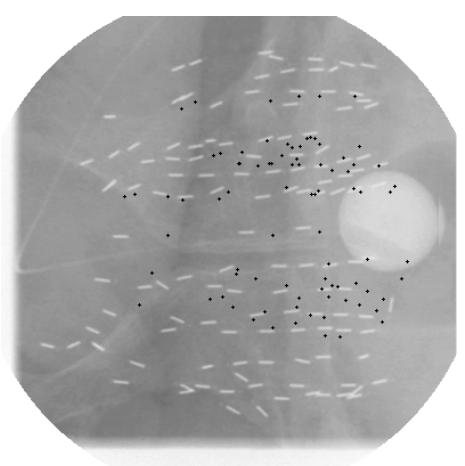

(a)

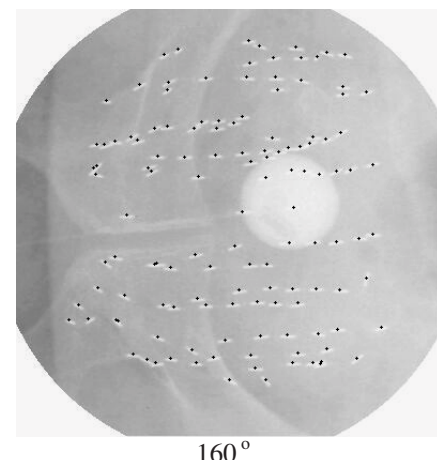

$160^{\circ}$

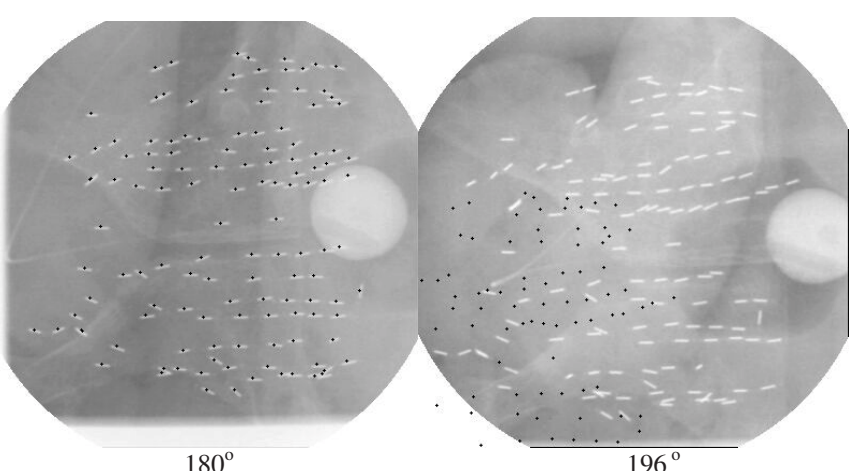

(b)

Figure 8. (a) A clinical example (128 implanted seeds) with large patient movement. Without motion correction, the reconstruction algorithm was not able to localize the seeds accurately. The reconstructed $3 \mathrm{D}$ seed coordinates were forward projected onto the $180^{\circ}$ view showing erroneous results. (b) The seed reconstruction followed by motion correction. The reconstructed seed centroids were forward projected onto each view demonstrating satisfactory reconstruction as verified on $160^{\circ}$ and $180^{\circ}$ views. The movement occurred between image captures for $180^{\circ}$ and $196^{\circ}$ angles.

The registration accuracy in terms of RMS error was $2.1 \pm 0.8 \mathrm{~mm}$. Figure 10 shows the superposition of the calculated seed coordinates on a CT slice.

\section{Discussion}

The simulation study was conducted in order to characterize the effect of motion correction on seed reconstruction accuracy. Increase in translational movement generally resulted in increase in reconstruction errors. It is noted that the errors in the $Z$-(anterior-posterior) direction are larger than those in $X$-(cranio-caudal) and $Y$-(lateral) directions. This is due to the fact that a given positional change in the $Z$-direction would manifest itself as a reduced, smaller shift in the projection data. Conversely, uncertainty in translating the projection data for movement correction is magnified in $3 \mathrm{D}$ space along the $Z$-direction. This effect is more pronounced when the angular separations between image captures are small. Errors in $X$ - and $Y$-directions are similar to each other and are an order of magnitude smaller than the $Z$ errors. As for the rotational movement, we have observed that since the patient's legs are supported by the stirrups at the knee joints, it would be difficult for the prostate to 


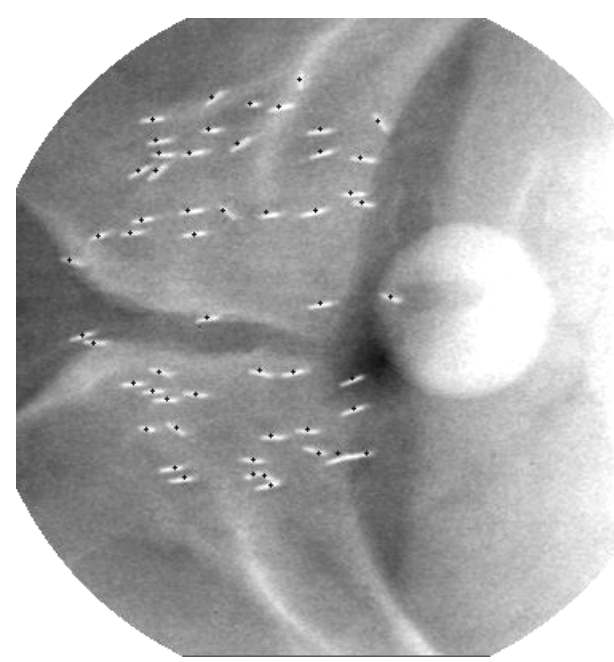

$171^{\circ}$

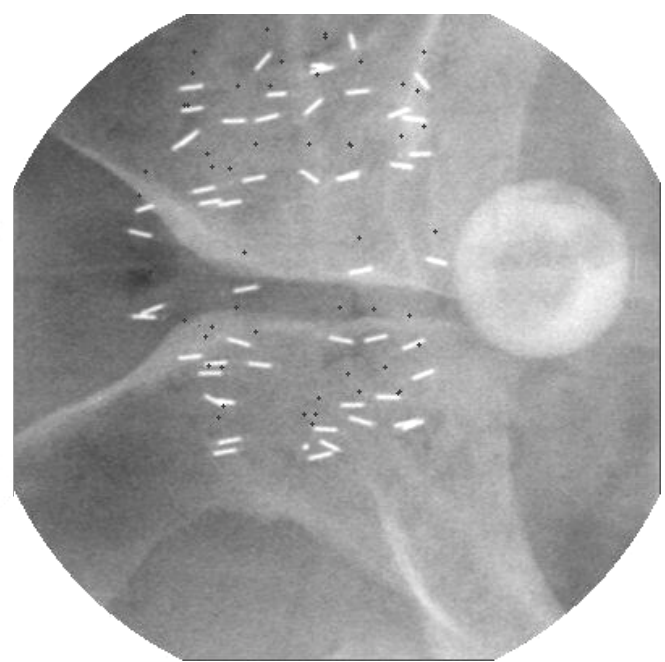

$180^{\circ}$

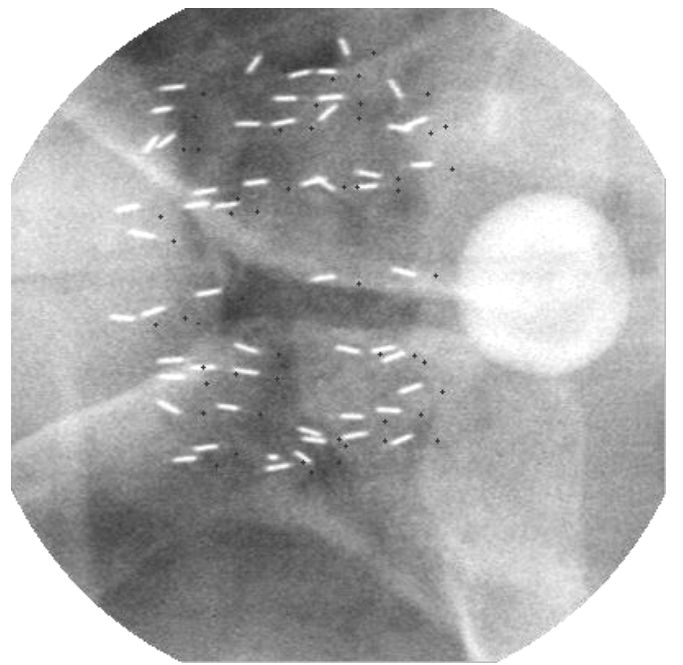

$189^{\circ}$

Figure 9. A clinical example with two separate movements: one between views $171^{\circ}$ and $180^{\circ}$ then another between views $171^{\circ}$ and $189^{\circ}$.

rotate about itself. If there is a rotational component to the movement, it is likely that the rotational axis is away from the prostate towards the legs. To simulate this scenario we have set the axis of rotation to be $15 \mathrm{~cm}$ from the prostate, which is approximately the distance from the prostate to the leg support along the cranio-caudal direction. For these simulations (cases \#2, 4 and 6), the errors were not much worse than those (cases \#1, 3 and 5) where the axis of rotation was at the centre of the prostate. This is because as the axis of rotation shifts farther away from the prostate, the rotational movement becomes more akin to a translational shift in $X$ - and $Y$-directions. Therefore, although the magnitude of the seed displacement due to movement increases for cases \#1, 3 and 5 compared to cases \#2, 4 and 6, the motion errors can be largely rectified by the $X$ and $Y$ correction methods, which are relatively more 


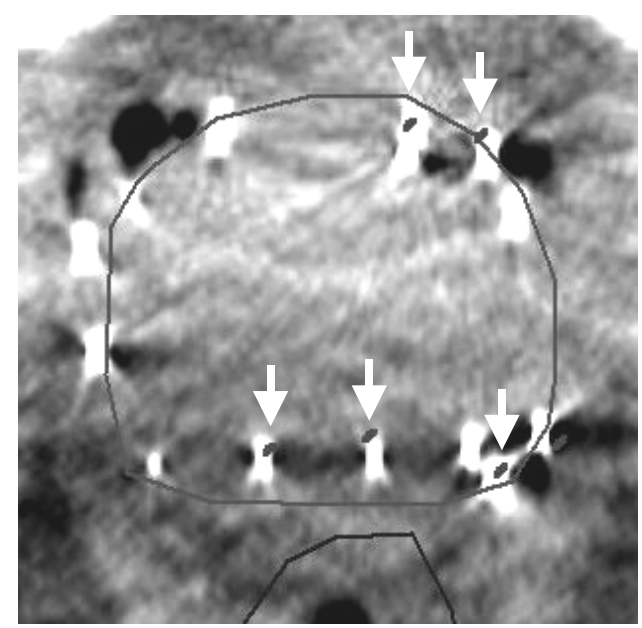

Figure 10. The seed coordinates calculated for the clinical example shown in figure 8 were registered with the CT-derived seed positions, and superposed on the CT images. Shown here is one of the CT slices with arrows pointing to the fluoroscopy-derived seed coordinates (marked by the black, slanted ellipses). CT slices were obtained at $3 \mathrm{~mm}$ increments. The fluoroscopy-derived seeds appear on the nearest slice only.

robust than the rotational correction algorithm. Rotational correction requires knowledge of the rotational-axis position, which is iteratively determined. However, the search method we have employed may not produce the best solution, as the strategy is susceptible to being trapped in a local minimum. Different combinations of rotational-axis position and angle may produce similar cost function values. The quadtree strategy does not exhaustively explore the search space and thus the cost associated with the final solution may not be the global minimum.

The patient movement during fluoroscopy image capture in our clinic has been found to be within $2 \mathrm{~cm}$ for the majority of cases. Therefore, the physical phantom was moved by $2 \mathrm{~cm}$ to examine the upper bound of the clinical patient movement range. The exercise included uncertainties introduced by the seed detection process and the results indicated RMS errors of $0.2,0.3$ and $0.6 \mathrm{~mm}$ in $X-, Y$ - and $Z$-directions, respectively. The corresponding standard deviations were $0.1,0.1$ and $0.4 \mathrm{~mm}$. The magnitude of errors should not significantly affect the dosimetric parameters. For example, the study by Lindsay et al (2003) indicates that the average changes in D90 and V90 are less than $2 \%$ when the seed localization errors are within the normal standard deviation of $1.5 \mathrm{~mm}$.

Two different clinical examples were presented. The first example (figure 8) illustrates a case where the patient movement is relatively large $(2.5$ and $2.4 \mathrm{~cm}$ in $X$ - and $Y$-directions). The second example (figure 9) demonstrates the ability of the algorithm to deal with two separate movements between image acquisitions. To date, we have successfully performed intraoperative dosimetry on 228 patient cases using our seed reconstruction algorithm coupled with the proposed motion correction technique. Of the 228 cases, patient movements were observed in 96 cases (42\%). Most of the cases ( 89 cases) involving motion correction had only one movement. There were only 7 cases (3\%) with two movements. These statistics indicate that where local anaesthesia is used the seed reconstruction algorithm must be able to handle patient movement. There were only a few cases of seed reconstruction failure due to large patient movement. In such cases, fluoroscopy imaging can be repeated. 
The eigen lines provide convenient reference for movement analysis along the axis perpendicular to the eigen plane, which, in our case, is along the $Y$-(lateral) direction. On the other hand, motion analysis in the direction parallel to the eigen plane (cranio-caudal or $X$-direction) is complicated by the lack of invariance in imaging geometry. This problem was overcome through indirect analysis of $X$-movement using the $Z$-component.

For each component, the general strategy was to correct for patient movement that has occurred in $3 \mathrm{D}$ space by manipulating the projection data in $2 \mathrm{D}$. This is an approximate method and does not exactly account for the spatial transformation due to conical projection geometry. However, when the extent of seed distribution is small relative to the distance between the x-ray source and the detector, the approximate approach can produce acceptable results.

The algorithm was coded in $\mathrm{C}++$ in Microsoft Windows environment. The computation time scales linearly with the number of seeds. The processing time for the 128 -seed clinical example was $5.5 \mathrm{~s}$ on a $\mathrm{PC}$ with a $2.8 \mathrm{GHz}$ Pentium 4 processor.

\section{Conclusion}

We have developed an algorithm that permits detection and correction of patient movement that may occur between fluoroscopy image captures. The patient movement is decomposed into translational shifts along the tabletop and rotation about an axis perpendicular to the tabletop. The property of spatial invariance of the co-planar imaging geometry is used for lateral movement correction. Cranio-caudal movement is corrected by analysing the perspective invariance along the x-ray axis. Rotation is estimated by an iterative method. The method can detect and correct for the range of patient movement commonly seen in the clinical environment. The algorithm has been implemented for routine clinical use as the preprocessing step for seed reconstruction.

\section{Acknowledgment}

This work was supported in part by a grant from the Department of Defense Prostate Cancer Research Program (DAMD17-03-1-0033).

\section{References}

Altschuler M D, Findlay P A and Epperson R D 1983 Rapid, accurate, three-dimensional location of multiple seeds in implant radiotherapy treatment planning Phys. Med. Biol. 28 1305-18

Altschuler M D and Kassaee A 1997 Automated matching of corresponding seed images for three simulator radiographs to allow 3D triangulation of implanted seeds Phys. Med. Biol. 42 293-302

Amols H I and Rosen I I 1981 A three-film technique for reconstruction radioactive seed implants Med. Phys. 8 210-4

Biggs P J and Kelly D M 1983 Geometric reconstruction of seed implants using a three-film technique Med. Phys. $10701-5$

Blasko J C, Ragde H, Luse R W, Sylvester J E, Cavanagh W and Grimm P D 1996 Should brachytherapy be considered a therapeutic option in localized prostate cancer? Urol. Clin. North Am. 23 633-50

Blasko J C, Ragde H and Schumacher D 1987 Transperineal percutaneous iodine-125 implantation for prostatic carcinoma using transrectal ultrasound and template guidance Endocurietherapy/Hyperthermia Oncol. 3 131-9

Cho P S 2000 Computerized segmentation of clustered seeds in prostate brachytherapy Proc. 13th Int. Conf. on Computers in Radiotherapy ed W Schlegel and T Bortfeld (Heidelberg: Springer) pp 105-7

Grimm P D, Blasko J C, Sylvester J E, Meier R M and Cavanagh W 2001 10-year biochemical (prostate-specific antigen) control of prostate cancer with I-125 brachytherapy Int. J. Radiat. Oncol. Biol. Phys. 51 31-40

Jackson D D 1983 An automatic method for localizing radioactive seeds in implant dosimetry Med. Phys. 10 370-2 
Lam S T, Cho P S, Marks R J II and Narayanan S 2004 3D Seed reconstruction for prostate brachytherapy using Hough trajectories Phys. Med. Biol. 49 557-69

Lindsay P E, Van Dyk J and Battista J J 2003 Imaging uncertainties in prostate brachytherapy Med.Phys. 30 1897-908

Narayanan S, Cho P S and Marks R J II 2002 Fast cross-projection algorithm for reconstruction of seeds in prostate brachytherapy Med. Phys. 29 1572-9

Narayanan S, Cho P S and Marks R J II 2004 3D seed reconstruction from incomplete data set in prostate brachytherapy Phys. Med. Biol. 49 3483-94

Rosenthal M S and Nath R 1983 An automatic seed identification technique for interstitial implants using three isocentric radiographs Med. Phys. $10475-9$

Siddon R L and Chin L M 1985 Two-film brachytherapy reconstruction algorithm Med. Phys. 12 77-83

Smathers S, Wallner K, Simpson S and Roof J 2000 Patient perception of local anesthesia for prostate brachytherapy Semin. Urol. Oncol. 18 142-6

Todor D A, Zaider M, Cohen G N, Worman M F and Zelefsky M J 2003 Intra-operative dynamic dosimetry for prostate implants Phys. Med. Biol. 48 1153-71

Tubic D, Zaccarin A, Beaulieu L and Pouliot J 2001 Automated seed detection and three-dimensional reconstruction: II. Reconstruction of permanent prostate implants using simulated annealing Med. Phys. 28 2272-9

Wallner K 2002 Prostate brachytherapy under local anesthesia; lessons from the first 600 patients Brachytherapy 1 $145-8$

Wallner K, Simpson C, Roof J, Arthurs S, Korssjoen T and Sutlief S 1999 Local anesthesia for prostate brachytherapy Int. J. Radiat. Oncol. Biol. Phys. 45 401-6 